\title{
Numerical Simulation of Solitary Waves by SPH Method and Parametric Studies on the Effect of Wave Height to Water Depth Ratio
}

\author{
Parviz Ghadimi, Shahryar Abtahi, Abbas Dashtimanesh \\ Department of Marine Technology, Amirkabir University of Technology, Tehran, \\ Iran \\ E-mail: pghadimi@aut.ac.ir, sh.abtahi@aut.ac.ir, $\underline{\text { a.dashtimanesh@aut.ac.ir }}$
}

\begin{abstract}
Numerical simulation of fluid flow phenomena, including complicated free surface deformation, has been a main research interest for many authors. In this context, using mesh based methods has been a common choice in the past decades. However, in the recent years, Smoothed Particle Hydrodynamics (SPH) as a Lagrangian meshless method has been utilized in many of applications. In the present article, a two dimensional solitary wave on a beach is generated and the effect of various wave heights to water depth ratios on solitary wave generation are studied. Furthermore, condition in which wave breaking occurs is also considered. Finally, the results of SPH simulations are compared against the available results in the literature and it is shown that Solitary wave profile simulated by SPH is in good agreement with experimental data.
\end{abstract}

Keywords: Smoothed Particle Hydrodynamics, Solitary Waves, Wave Breaking, Symplectic Algorithm, Cubic Spline Kernel Function

\section{Introduction}

Interaction of solitary wave and marine structures and run up of solitary wave on slope beaches have always been a fundamental problem in ocean engineering. Furthermore, study of solitary wave interaction with the slope beaches has been the center of attention, over the years. In the beach zones where the water depth is shallow, solitary wave is strongly influenced by the bed slope [1]. Since experimental modeling of solitary waves is very expensive, the numerical modeling is wave can be a good alternative. 
So far, various numerical methods for simulation of free surface flow have been implemented. Grilli and Seveden [2] used high order boundary element method (BEM) to simulate nonlinear water waves. Grilli [3] also developed a numerical model for nonlinear wave propagation in the physical space. He used a higher order BEM for solving Laplace's equation with higher order Taylor expansion for time integration of the two nonlinear free surface boundary conditions. Finally, he could simulate solitary wave with a reasonable accuracy. Furthermore, Grilli et al. [4] studied the characteristic of solitary wave such as wave length, wave velocity, wave height to water depth ratio $(\mathrm{H} / \mathrm{d})$, and breaking of the solitary wave. There also exist some studies which have focused on the interaction of solitary wave on slope beaches with inclined and vertical wall.

A numerical method was developed by Fenton and Rienecher [5] for solution of the fully nonlinear equations governing the irrotational flow with free surface and variable bed topography. This method was applied to analyze the unsteady motion of non-breaking water waves of arbitrary magnitude over a horizontal bed. All horizontal variation was approximated by truncated Fourier series. For nonbreaking waves the method was capable of performing high accuracy computations. Sen [6] used cubic spline variable and by assuming variable boundaries, extended high order integral equation method. For a large number of problems where the solution is sought in time domain, the (BIEM) solver, used as the field equation solver, must be applied at every time instant. For such a time domain simulation algorithm, the accuracy of (BIEM) solution is extremely important due the fact that, if even a small error exists, numerical instability may be introduced. According to Sen [7], accuracy of (BIEM) solver was improved by reduction of numerical errors on the interface.

In recent years, several researchers have tried to develop the next generation of numerical methods (Meshless Particle Methods) which are expected to be superior. In the last decade, some mesh free methods have been invented for analyzing the free surface phenomena. Smoothed Particle Hydrodynamics (SPH), as a meshless particle method, which was originally used to simulate astrophysical phenomena may be mentioned as the most famous meshless method to model the free surface flows.

Recently, Crespo et al. [8] proposed a kernel particle method which leads to accurate solution near the boundaries. Simulation of solitary wave on Carten beach was performed by Monaghan and Kos [9]. They simulated run up and wave breaking in laboratory and compared it with the SPH results. Loa and Shao [10], by combining SPH and LES, simulated solitary wave in vicinity of slop and shallow beaches. They solved Lagrangian form of the incompressible NavierStokes equations. Breaking of solitary waves on slope and shallow beaches were also simulated. Furthermore, Dalrymple and Rogers [11] simulated solitary waves. They considered different algorithms of filter density and viscosity, and several time stepping algorithms were implemented by them.

In the present article, SPH is applied to generate solitary wave. Wave generation is held in a numerical wave tank by a piston with prescribed motion. The wave 
maker is located on the left side of a wave tank and by generating solitary wave, it moves from left side to the right side of the tank and reaches the inclined side of tank which has a slope similar to the beaches. In a certain domain, seven solitary waves with wave heights to water depth ratios $(\mathrm{H} / \mathrm{d})$ of 0.1 to 0.7 are investigated. Breaking of solitary wave is also investigated for the ratios $\mathrm{H} / \mathrm{d}=0.794$ and 2 , which satisfy the Mitch condition. Generally, the main novelty of the present article is usage of SPH to study the solitary wave characteristics at different H/d ratios and the condition in which wave breaking occurs. Moreover, to improve the SPH results, the Shepard density filter is implemented in the current study.

\section{Fundamentals of SPH}

Smoothed Particle Hydrodynamics is a numerical method with Lagrangian nature. The governing differential equations contain $\rho$ (density), $\mathrm{V}$ (velocity), $\mathrm{r}$ (space vector) and $\mathrm{P}$ (pressure tensor) as [12]:

$$
\begin{gathered}
\frac{d P}{d t}=-\rho \nabla \cdot V \\
\frac{d V}{d t}=\frac{1}{\rho} \nabla \cdot P
\end{gathered}
$$

In the SPH method, each function $\mathrm{f}\left(\mathrm{r}_{\mathrm{i}}\right)$ can be approximated as follows:

$f\left(r_{i}\right)=\sum_{j} m_{j} \frac{f_{j}}{\rho_{j}} W\left(r_{i}-r_{j}\right)$

$\mathrm{P}, \rho, \mathrm{V}$ are interpolated using a kernel function [13]. In the above equation, $f_{j}$ and $m_{j}$ are the particle value and mass at particle $j$, respectively. Also, a derivative of function $f$ is discretized by

$\nabla f\left(r_{i}\right)=\sum m_{j} \frac{f_{j}}{\rho_{j}} \nabla W\left(r_{i}-r_{j}\right)$

In these equations, $W$ is the kernel function.

\subsection{Kernel Function Characteristics}

Numerical simulation by the SPH method strongly depends on the Kernel function. In the current work, cubic spline kernel function is selected [13] as in 
$w(r, h)=\alpha_{D}\left\{\begin{array}{cc}1-\frac{3}{2} q^{2}+\frac{3}{4} q^{3} & 0 \leq q \leq 1 \\ \frac{1}{4}(2-q)^{3} & 1 \leq q \leq 2 \\ 0 & q \geq 2\end{array}\right.$

where $\alpha_{D}$ is $\left(\frac{10}{\pi h^{2}}\right)$, in two dimensions simulations and $h$ denotes the smoothing length.

\subsection{Density Approximation}

For obtaining the discrete form of $\rho_{i}$, it is possible to replace $\rho_{i}$ by $f_{i}[12]$ as in

$\rho_{i}=\sum_{j} m_{j} W_{i j}$

Furthermore, implementing the density filter causes the results to improve. Therefore, Shepard density filter is used in the present study. This filter is a rapid and simple density corrector. It is utilized in every thirty steps, and the particle density is revised [14]:

$\rho_{i}^{\text {new }}=\sum_{j} \rho_{j} W_{i j} \cdot \frac{m_{j}}{\rho_{j}}=\sum_{j} m_{j} W_{i j}$

\subsection{Approximation of Navier Stokes Equation in SPH Notation}

To obtain a computational model for the particle's motion, pressure gradient tensor $\nabla\left(\frac{P}{\rho}\right)$ should be calculated [12]:

$$
\begin{gathered}
\nabla \cdot \frac{P}{\rho}=-\frac{P}{\rho^{2}} \cdot \nabla \cdot \rho+\frac{1}{\rho} \cdot \nabla \cdot P \quad \text { or } \quad-\frac{1}{\rho} \cdot \nabla \cdot P \\
=-\nabla \cdot \frac{P}{\rho}-\frac{P}{\rho^{2}} \cdot \nabla \cdot \rho
\end{gathered}
$$

Substituting this relation into equation 2 would yield in

$$
\begin{aligned}
& \frac{d V}{d t}=-\frac{P}{\rho^{2}} \cdot \nabla \cdot \rho-\nabla \cdot \frac{P}{\rho} \\
& \nabla \rho=\sum_{j} m_{j} \nabla_{j} W_{i j}
\end{aligned}
$$


$\nabla \cdot \frac{P}{\rho}=\sum_{j} m_{j} \cdot \frac{P_{j}}{\rho_{j}^{2}} \cdot \nabla_{j} W_{i j}$

Subsequently, equation of motion of particle becomes

$$
\begin{aligned}
\frac{d V_{i}}{d t}=-\frac{P_{i}}{\rho_{i}^{2}} \sum_{j} & m_{j} \nabla_{j} W_{i j} \\
& -\sum_{j} m_{j} \frac{P_{j}}{\rho_{j}^{2}} \nabla_{j} . W_{i j}
\end{aligned}
$$

Continuity equation (1) which contains the velocity gradient is also discretized as follows[12]:

$$
\begin{aligned}
& \frac{d \rho}{d t}=-\rho \nabla . V \\
& \nabla(V \rho)=\rho \nabla V+V \nabla \rho .
\end{aligned}
$$

Substitution of equation 6 into this equation would lead to

$$
\begin{aligned}
& \frac{d \rho}{d t}=-\nabla(V \rho)+V \nabla \rho, \\
& \frac{d \rho_{i}}{d t}=-\sum_{j} m_{j} V_{i j} \nabla_{j} W_{i j} .
\end{aligned}
$$

\subsection{Boundary Conditions}

In this work, dynamic boundary condition [13] is applied. Boundary particles have the same behavior as the fluid particles and follow continuity, momentum and state equations. Contrary to a fluid particle which has displacement, the displacements of boundary particles are zero. Arrangement of boundary particles is displayed in Fig.1.

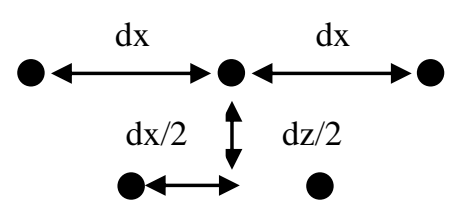

Fig.1. Interaction between fluid particles and boundary particles.

Based on Eq.10, while a particle reaches the boundaries, density of boundary particles are increased. Because of the pressure term $(\mathrm{P} / \rho)$ in momentum equation, the force which acts on the fluid particle increases. When the distance between 
boundary particle and fluid particle decreases, density, pressure and acting force on the fluid particle increase by a repulsive mechanism. More details can be found in reference [13].

\subsection{Artificial Viscosity}

Artificial viscosity was introduced by Monoghan [14] and applied to SPH formulation, due to its simplicity. Based on the SPH formulation, we have

$\frac{d v_{a}}{d t}=-\sum_{b} m_{b}\left(\frac{P_{a}}{P_{a}^{2}}+\frac{P_{b}}{P_{b}^{2}}\right.$

in which pressure gradient and $\Pi_{\mathrm{ab}}$ are

$$
\begin{aligned}
& -\frac{1}{\rho} \nabla P=-\sum_{b} m_{b}\left(\frac{P_{a}}{P_{a}^{2}}+\frac{P_{b}}{P_{b}^{2}}\right) \nabla a W_{a b} \\
& \Pi_{a b}= \begin{cases}\frac{-\alpha \overline{C_{a b}} \mu_{a b}}{\overline{\rho_{a b}}} & V_{a b} \cdot r_{a b}<0, \\
0 & \text { for other values }\end{cases}
\end{aligned}
$$

where $\bar{\rho}_{\mathrm{ab}}$ and $\mu_{\mathrm{ab}}$ are:

$\mu_{a b}=\frac{h v_{a b} \cdot r_{a b}}{r_{a b}^{2}+\eta^{2}}$

$\bar{\rho}_{a b}=\frac{1}{2}\left(\rho_{a}+\rho_{b}\right), \bar{C}_{a b}=\frac{1}{2}\left(C_{a}+C_{b}\right) ; \eta^{2}=0.01 h^{2}$

The value of $\alpha$ can be varied based on the type of problem.

\section{Wave Paddle}

Generation of solitary wave by a piston wave maker is performed using first order theory of solitary wave [15]. Displacement of paddle is based on the following equation [15]:

$X_{p}(t)=\frac{H}{k}\left[\tanh (X(t))+\tanh \left(\frac{k}{d} \lambda\right)\right] ; \quad X(t)=\frac{k}{d}\left(c t-X_{p}(t)-\lambda\right)$

where $\mathrm{H}$ is wave height, $k=\sqrt{\left(\frac{3 H}{4 d}\right)}$ is wave number and $c=\sqrt{g(d+H)}$ is velocity. Velocity of wave paddle can also be calculated by [15]:

$u_{p}(t)=\frac{c H}{d} \cdot \frac{1}{\cosh ^{2} X(t)+\frac{H}{d}}$ 


\section{Discussion of Results}

In the initial modeling, based on Sangita's [1] experiment, the length and flat length of the wave tank, water depth and slope of the right side of the tank were considered to be $10 \mathrm{~m}, 9.7 \mathrm{~m}, 0.3 \mathrm{~m}$ and $45 \mathrm{deg}$, respectively (as shown in Fig.2). Wave paddle motions for different $\mathrm{H} / \mathrm{d}$ ratios are calculated, as presented in table 1.

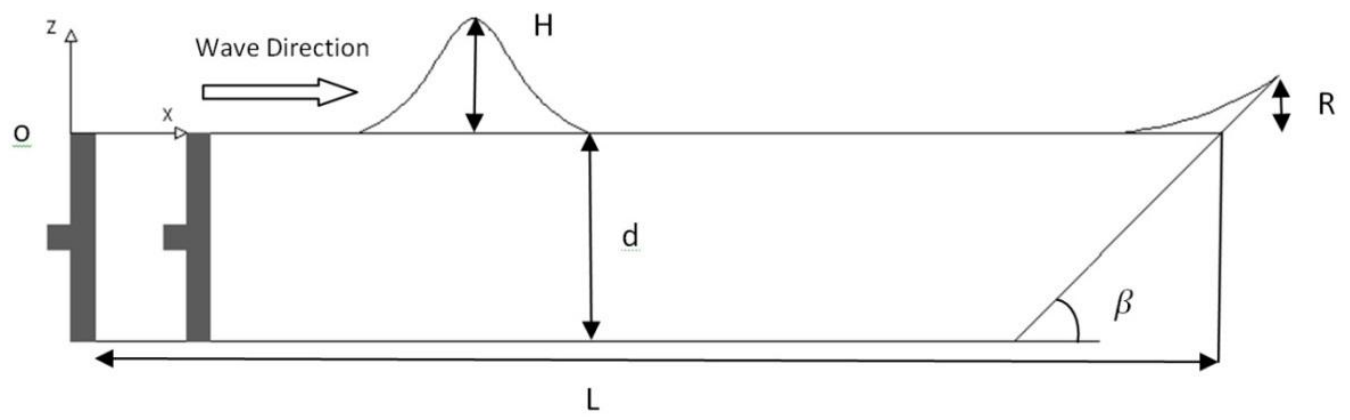

Fig.2. Computational Domain.

Table 1: Characteristics of the generated solitary waves.

\begin{tabular}{|c|c|c|c|c|c|c|c|}
\hline & 1 & 2 & 3 & 4 & 5 & 6 & 7 \\
\hline $\mathrm{H} / \mathrm{d}$ & 0.1 & 0.2 & 0.3 & 0.4 & 0.5 & 0.6 & 0.7 \\
\hline $\mathrm{H}$ & 0.03 & 0.06 & 0.09 & 0.12 & 0.15 & 0.18 & 0.21 \\
\hline $\mathrm{d}$ & 0.3 & 0.3 & 0.3 & 0.3 & 0.3 & 0.3 & 0.3 \\
\hline & 6.882 & 4.866 & 3.973 & 3.441 & 3.078 & 2.809 & 2.601 \\
\hline
\end{tabular}

The results of numerical simulation by SPH are compared to Sangita's [1] findings in Figs.3 through 9. Figure 3 shows the wave height of $0.03 \mathrm{~m}$. The computed results display high accuracy in the first 3 seconds and also between 9 and 10 seconds. Additionally, the wave crest matches very well with Sangita's [1] result.

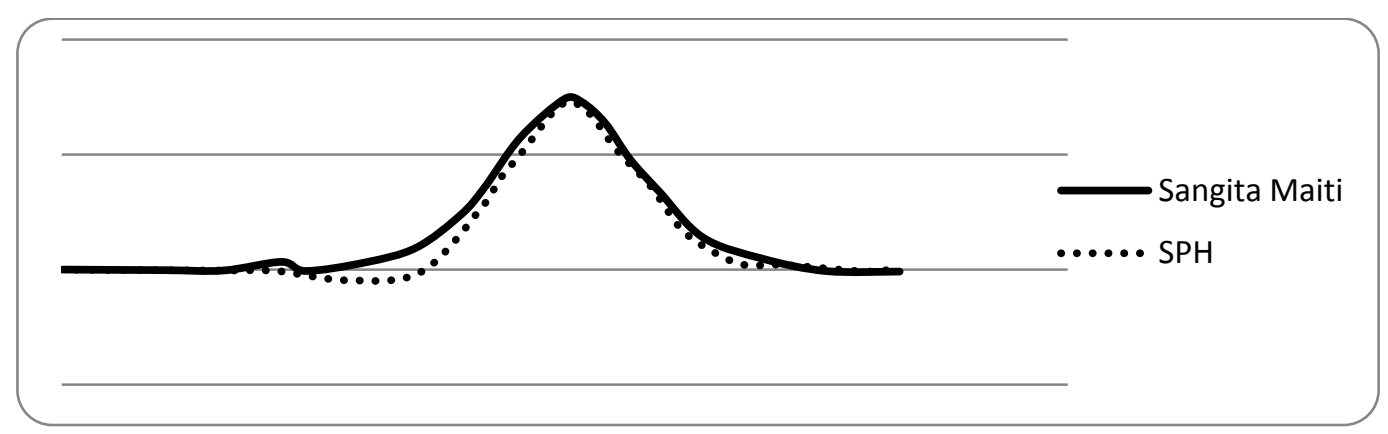

Fig.3. Comparison of solitary wave by Sangita's results $(\mathrm{H} / \mathrm{d}=0.1)[1]$. 
In figure 4, the water depth is $0.3 \mathrm{~m}$ and wave height is $0.06 \mathrm{~m}$. Generally, there is a 5 percent difference in the entire wave simulation and 2.5 percent difference in wave crest in comparison with Sangita's [1] experiment. Also, there exist some inconsistencies at $\mathrm{t}=4 \mathrm{~s}$ which may be due to the limitation in implementation of more particle's number.

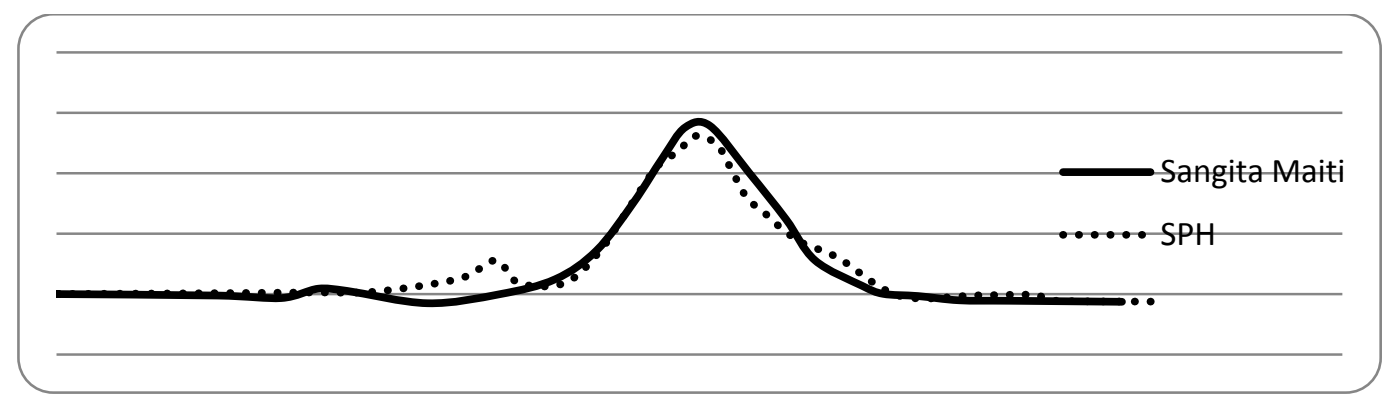

Fig.4. Comparison solitary wave by Sangita's results $(\mathrm{H} / \mathrm{d}=0.2)[1]$.

For the cases $\mathrm{H} / \mathrm{d}=0.3$ and 0.4 , simulations display great match with Sangita's simulations (as seen in Figs.5 and 6). For the time interval $t=3 \mathrm{~s}$ to $\mathrm{t}=5 \mathrm{~s}$, there exists small difference between the SPH results and Sangita's [1] findings, but at other time instants, SPH results demonstrate great accuracy.

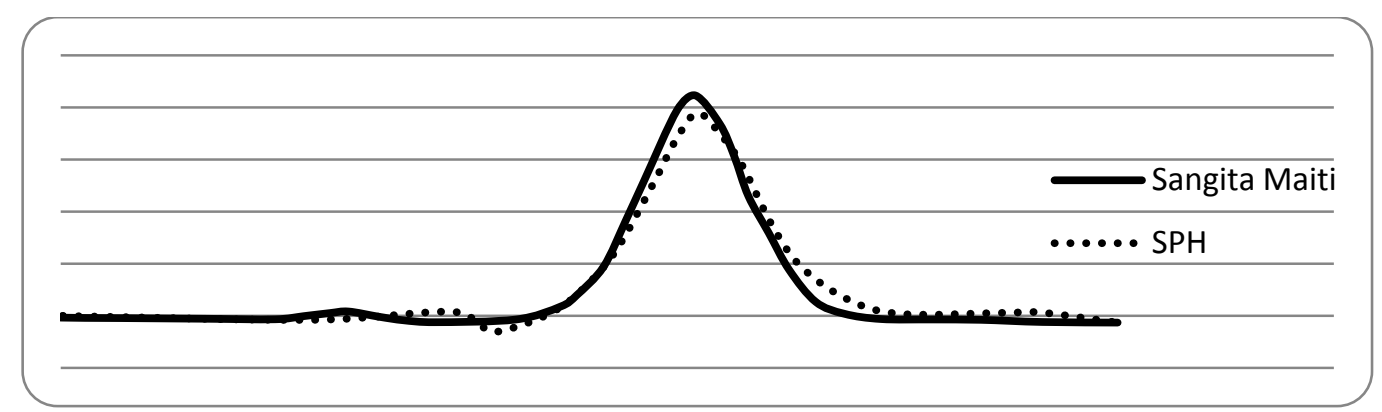

Fig.5. Comparison of solitary wave by Sangita's results $(\mathrm{H} / \mathrm{d}=0.3)[1]$.

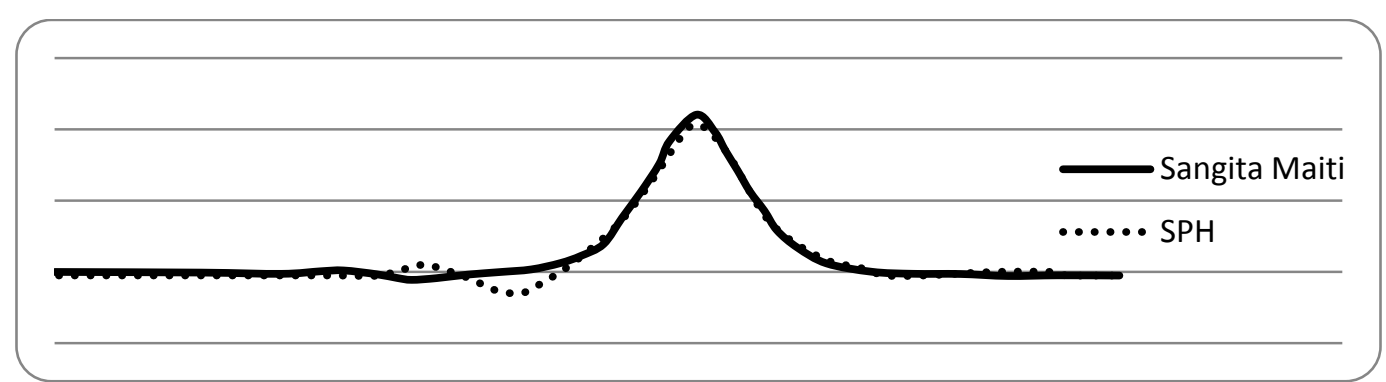

Fig.6. Comparison of solitary wave by Sangita's results $(\mathrm{H} / \mathrm{d}=0.4)[1]$.

Figures 7, 8 and 9 present the wave profiles for the cases $H / d=0.5,06$, and 07 , respectively. As evidenced in these figures, the numerical findings are in good 
agreement with Sangita's results [1]. The wave crest of all three cases coincided with Sangita's model, accurately. In these conditions, wave separates from the wave paddle and subsequently starts to develop and reach the designed wave height. The wave interacts with the slope beach region and wave run-up will appear.

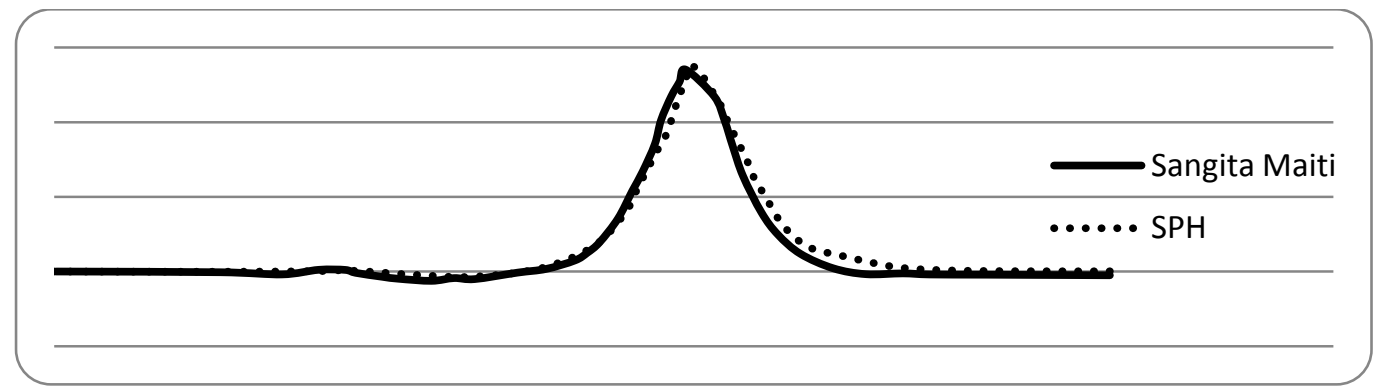

Fig.7. Comparison of solitary wave by Sangita's results $(\mathrm{H} / \mathrm{d}=0.5)[1]$.

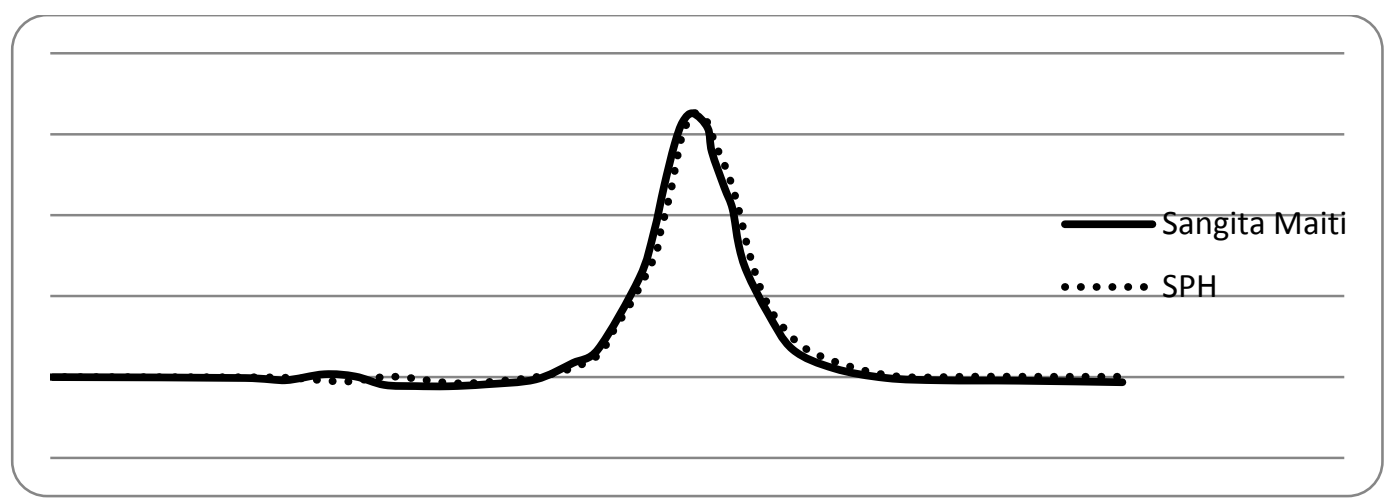

Fig.8. Comparison of solitary wave by Sangita's results $(\mathrm{H} / \mathrm{d}=0.6)[1]$.

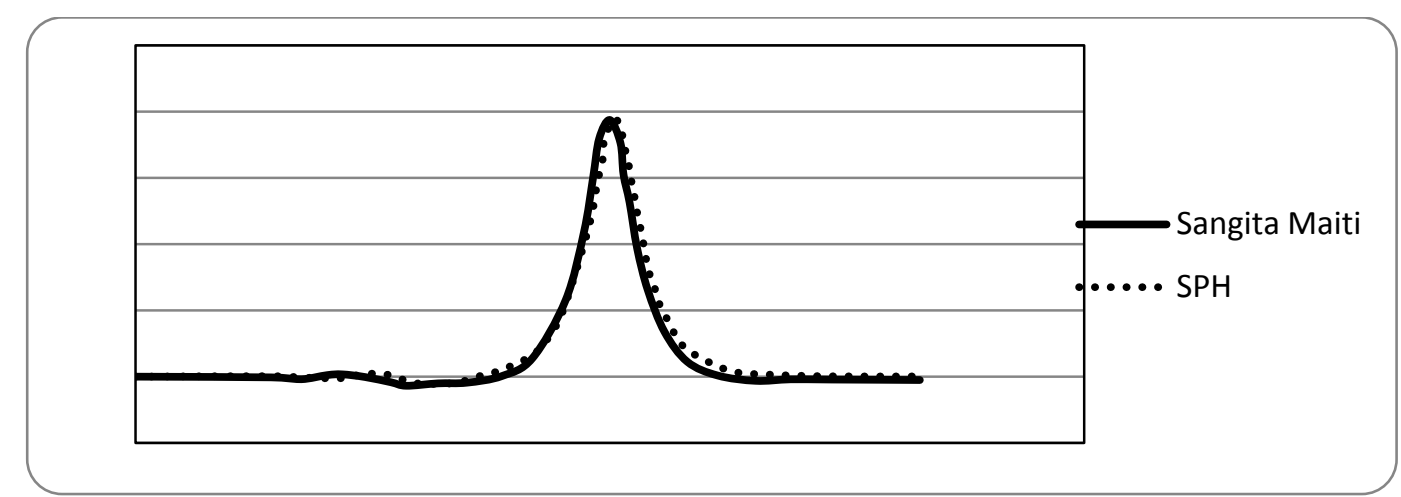

Fig.9. Comparison of Solitary wave by Sangita's results $(\mathrm{H} / \mathrm{d}=0.7)[1]$.

In the presented simulations, solitary waves have not broken. It is due to this fact that the Mitch conditions were not satisfied. Based on the Mitch conditions, a wave can break when the ratio of wave height to water depth $(\mathrm{H} / \mathrm{d})$ is more than 
0.7. Therefore, in subsequent simulations, two $\mathrm{H} / \mathrm{d}$ ratios of 0.794 and 2 will be investigated. In these circumstances, it is possible to see that solitary waves break on a slope beach. The wave tank at the ratio $\mathrm{H} / \mathrm{d}=0.794$ has a flat length of 2 meters and the degree of slope is 5.71 degrees (as seen in Fig.10). Figure 11 illustrates the result of this simulation.

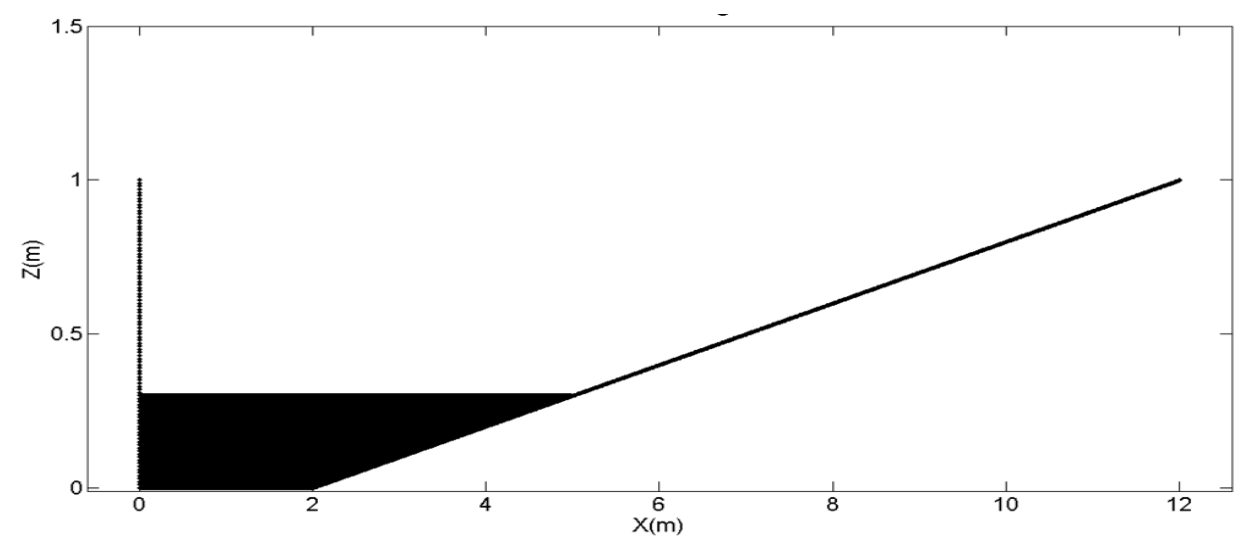

Fig.10. Computational Domain $(\mathrm{H} / \mathrm{d}=0.794)$.

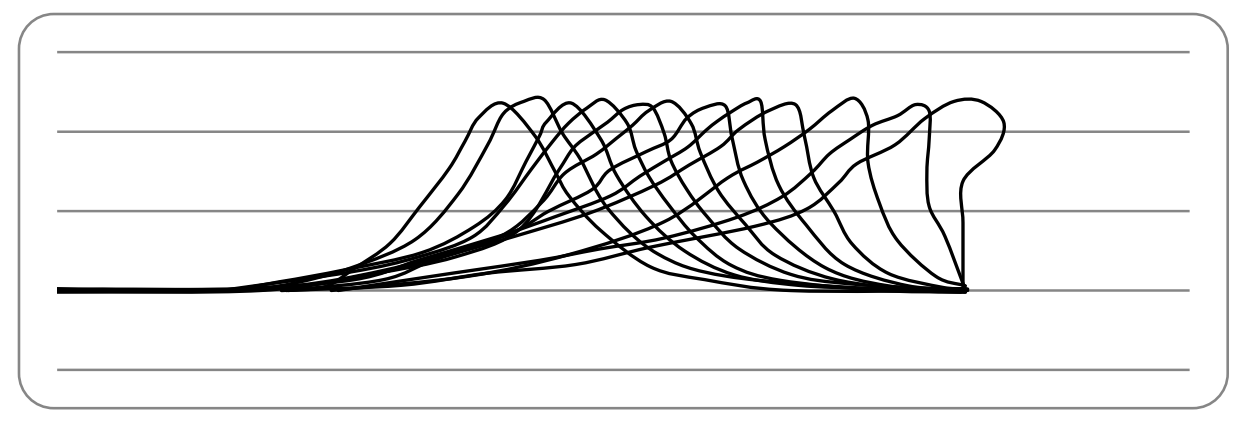

Fig.11. Process of breaking Wave formation $(\mathrm{H} / \mathrm{d}=0.794)$.

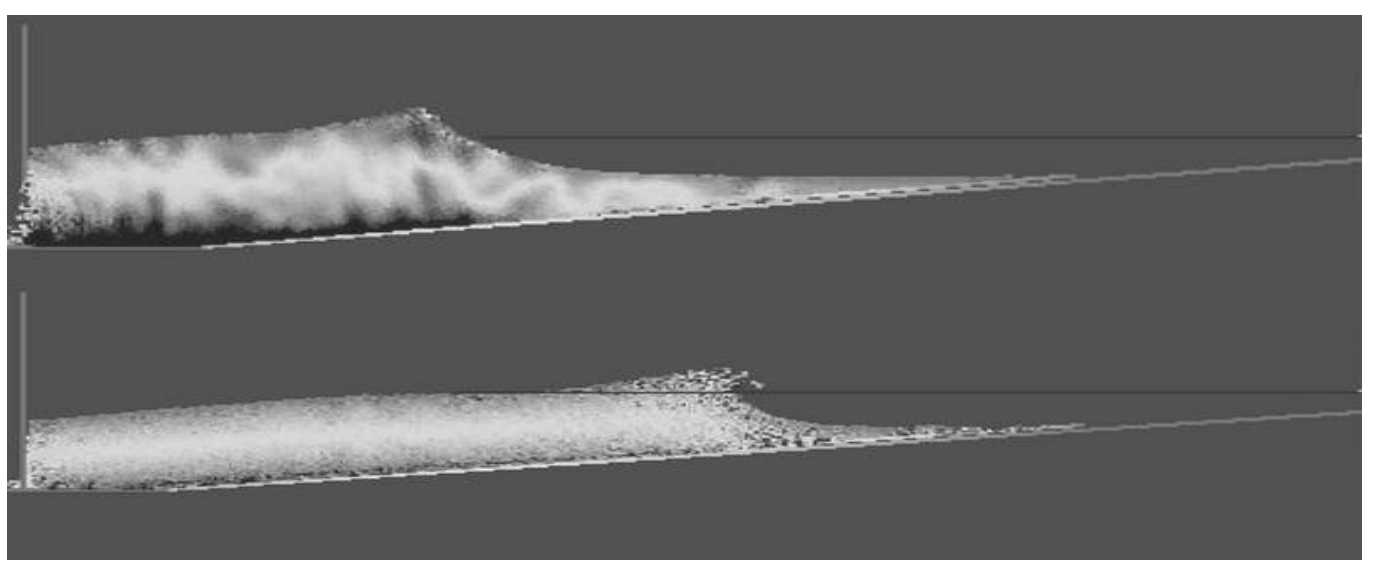

Fig.12. Numerical Simulation of breaking wave on slope beach with inclination angle of 5.41 degree $(\mathrm{H} / \mathrm{d}=0.794)$. 
It can be observed from Figs. 10 and 11 that a solitary wave is generated 2 meters from the location of the wave maker. The solitary wave moves forward and starts breaking. In this simulation, Mitch condition is satisfied and the solitary wave breaks at $\mathrm{x}=4 \mathrm{~m}$. Figure 12 graphically shows the breaking of the solitary wave.

Finally, numerical simulation of solitary wave is considered where $H / d=2$. In this numerical model, the wave tank's flat length is 1 meter and the slope of the inclined side is 5.71 degrees (as evidenced in Fig.13). Figure 14 shows the results of the solitary wave breaking when $\mathrm{H} / \mathrm{d}=2$.

In the last simulation $(\mathrm{H} / \mathrm{d}=2)$, the solitary wave height, over the spatial interval of $\mathrm{x}=1.4 \mathrm{~m}$ to $1.6 \mathrm{~m}$, reaches the height $0.6 \mathrm{~m}$ and rapidly starts breaking due to Mitch condition satisfaction. When the paddle moves and the wave reaches the $0.6 \mathrm{~m}$ height, the breaking of solitary wave is observed. Wave run up on the inclined side of the wave tank can also be seen. The run up is also observed which may be due to the incident of the solitary wave. Therefore, the maximum run up through our simulations can occur.

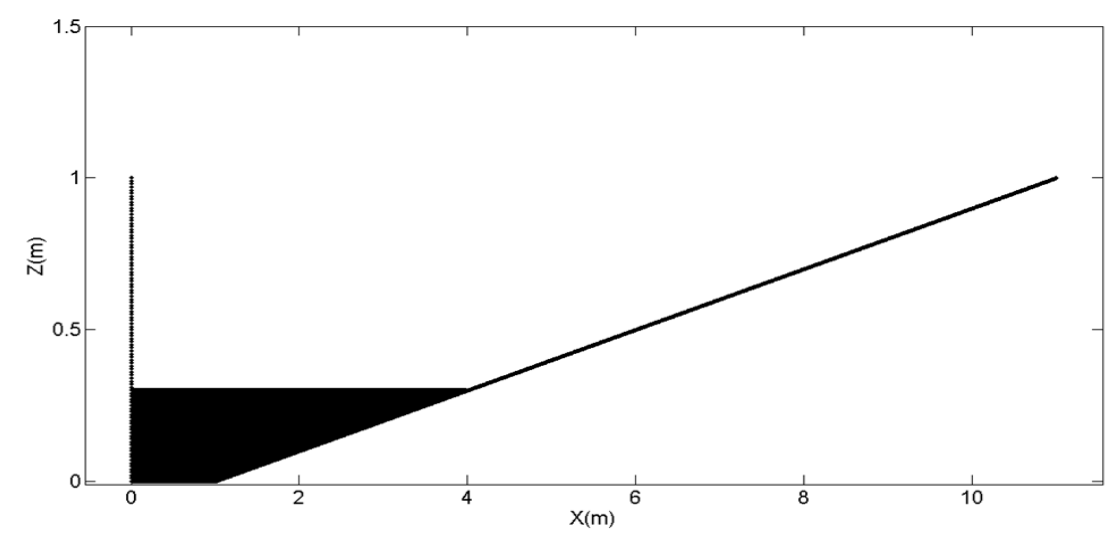

Fig.13. Computational Domain $(\mathrm{H} / \mathrm{d}=2)$.

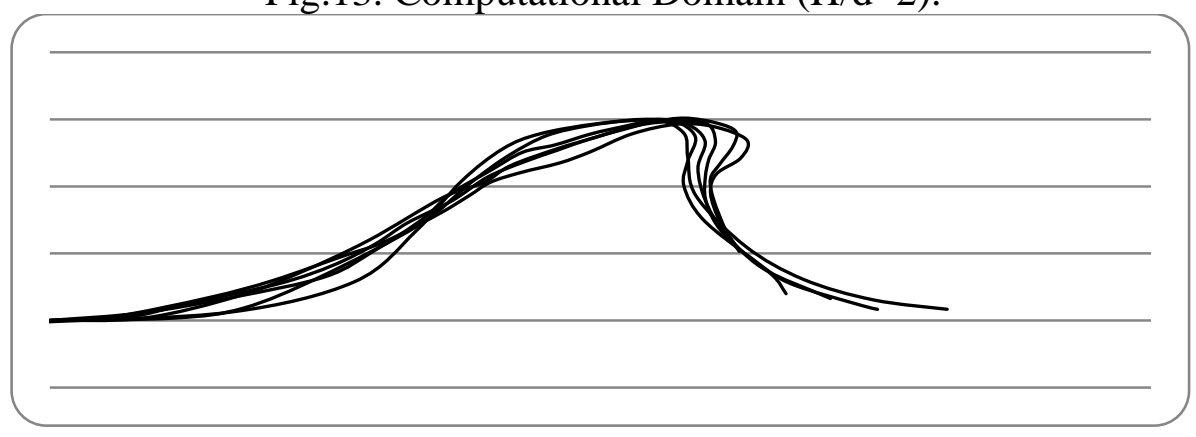

Fig.14. Breaking of Wave $(\mathrm{H} / \mathrm{d}=2)$. 


\section{Conclusion}

In the present study, solitary wave is simulated by SPH method at different wave height to water depth $(\mathrm{H} / \mathrm{d})$ ratios. Generating and breaking of the solitary wave is very sensitive to the number of particles, which basically means, the more particles the more accuracy. Cubic Spline is considered as a kernel function. Artificial viscosity was also applied and a filter density scheme is utilized to improve the computations.

After simulating the solitary wave which travels to the end of the wave tank and pours on the slope in non-breaking conditions, breaking of the solitary wave was also simulated. It was observed that satisfying Mitch condition, at $\mathrm{H} / \mathrm{d}=0.794$ and 2 , can cause breaking of the solitary wave on the inclined side of the wave tank. Comparison of the SPH result with that of Sangita's [1] finding shows good accuracy of the present simulations. Some inconsistencies which were detected at $\mathrm{t}=4 \mathrm{~s}$ of the simulation can probably be improved by increasing the number of particles. Overall, it is illustrated that the SPH simulation of the solitary wave has great accuracy in comparison with the experimental data.

Calculating wave force acting on marine structures is among engineers' concerns. Therefore, SPH modeling of solitary waves interacting with the marine structures and calculating the force for breakwaters, platforms among others are suggested as future studies.

\section{References}

[1] Sangita, M., "Computation of Solitary Waves During Propagation and Runup on a Slope", J. Ocean Engineering, Vol.26, No.11, (1999), pp.1063-1083.

[2] Grilli, S.T. and I.A. Seveden, "Computation of nonlinear wave kinematics during propagatopn and run-up on a slope", NATO Workshop, Model, Norway, (1990).

[3] Grilli, S.T. and I.A. Seveden, Corner problems and global accuracy in the boundary element solution of nonlinear water waves, Engineering Analysis with Boundary Elements, Vol.7, No.4, (1990), pp.178-195.

[4] Grilli, S.T. and I.A. Seveden, R. Subramanya,"Breaking criterion and characteristics for solitary", Journal of Waterway Port Coastal and Ocean Engineering, Vol.123, No.3, (1997), pp. 102-112.

[5] Fenton, J.D. adn M.M. Rienecher, "A Fourier Method for Solving nonlinear Water-Wave Problems: Application to Solitary Wave Interactions", Journal of Fluid Mechanics, Vol.118, (1982), pp.411-443.

[6] Sen, D., "A cubic spline integral method for two-dimensional free-surface flow problems", International Journal for Numerical Methods in Engineering, Vol.38, (1995), pp.1809-1830. 
[7] Sen, D., "An accurate cubic-spline boundary integral formulation for twodimensional free-surface flow problems", Lecture Notes in Physics, Springer, Berlin, Vol.453, (1995), pp.216-220.

[8] Crespo, A., "Application of the smoothed particle hydrodynamics model SPHysics to free surface hydrodynamics", Ph.D. thesis, University of De Vigo, (2008).

[9] Monaghan, J. J. and A. Kos, "Solitary Waves on a Cretan Beach", J. Waterway, Port, Coastal and Ocean Engineering, Vol.125, (1999), pp.145154.

[10] Loa, Y.M. and S. Shao, "Simulation of near-shore solitary wave mechanics by an incompressible SPH method", Applied Ocean Research,Vol.24, No.5, (2002), pp.275-286.

[11] Dalrymple, R.A. and B.D. Rogers., Numerical modeling of water waves with the SPH method, Coastal Engineering, Vol.53, No (2-3), (2005), pp.141147.

[12] Georg, C., "The Implementation of Smooth Particle Hydrodynamics in LAMMPS", Ph.D. thesis, University of Leuven, (2011).

[13] Liu, G.R. and M.B. Liu, "Smoothed particle hydrodynamics: a mesh free particle method", World Scientific, Singapore, (2003).

[14] Monaghan, J.J., "Simulating free surface flows with SPH, Journal Computational Physics", Vol.110, (1994), pp.399-406.

[15] Goring, D.G., "Tsunamis - the propagation of long waves onto a shelf", W. M. Keck Laboratory of Hydraulics and Water Resources, California Institute of Technology, Pasadena, CA., Report No. KH-R-38, (1978), 337. 